\title{
Surface-Only Spectroscopy for Diffusion-Limited Systems Using UI- tra-Low Temperature DNP MAS NMR at 16.4 T
}

\author{
Yoh Matsuki, ${ }^{\dagger}$ Tomoaki Sugishita, ${ }^{\dagger}$ Toshimichi Fujiwara ${ }^{\dagger}{ }^{*}$ \\ †Institute for Protein Research, Osaka University, Suita, Osaka 565-9871, Japan \\ ${ }^{\perp}$ Center for Quantum Information and Quantum Biology, Institute for Open and Transdisciplinary Research Initiatives, \\ Osaka University, Suita, Osaka 565-9871, Japan \\ Dynamic Nuclear Polarization, Ultra-Low Temperature, Closed-Cycle Helium MAS, Surface-Only Spectroscopy
}

\begin{abstract}
Conventional dynamic-nuclear-polarization (DNP) technique at $T \sim 100 \mathrm{~K}$ can enhance sensitivity of magic-angle spinning (MAS) NMR over 100-fold for standard samples containing urea/proline at high-field conditions, $B_{0}=9.4-16.4 \mathrm{~T}$. In the scene of real applications, however, the achievable enhancement is often much lower than for urea/proline due to faster ${ }^{1} \mathrm{H}$ relaxation $\left(T_{1 \mathrm{H}}\right)$ promoted by molecular-segmental fluctuations and methyl-group rotations active even at low temperatures, hindering an efficient polarization diffusion within the system. Here, we show at $16.4 \mathrm{~T}$ that ultra-low temperature $(T \ll 100 \mathrm{~K})$ provides a general way to improve the DNP efficiency for such diffusion-limited systems as we demonstrate on microcrystalline sample of a tripeptide $\mathrm{N}-f-\mathrm{MLF}-\mathrm{OH}$. In a further step, the hyperpolarization localized at the crystal surface enabled "surface-only" spectroscopy eliminating background signals from the crystal core. The surface-only data, rather than the currently popular surface-enhanced data, should prove to be useful in many applications in biological and material sciences.
\end{abstract}

Recent advent of the high-field dynamic nuclear polarization (DNP) technique has dramatically increased the sensitivity of magic-angle spinning (MAS) NMR, and widened the research scopes in various fields including chemistry, biology and material sciences. ${ }^{1}$ The NMR signal enhancement by the cross effect-based DNP observed for small-molecule (urea/proline) solution samples for benchmarking purpose often exceed a factor of 100 even at relatively high external-field conditions $B_{0}=9.4$ $-16.4 \mathrm{~T}$ and $T \sim 100 \mathrm{~K} .{ }^{2,3}$ In the scene of real applications, however, the achievable enhancement is generally much lower even after sample optimization for the choice of radical, its concentration and the ${ }^{1} \mathrm{H}$ content in the matrix etc. This is presumably because of much faster nuclear longitudinal relaxation $\left(T_{1}\right)$ in those "real" systems due to sidechain-dihedral/ring fluctuations and methyl-group rotation active even at very low temperatures; for polarizing a bulk sample by DNP, the polarization needs to be transferred from the radical to nearby nuclei (usually ${ }^{1} \mathrm{Hs}$ ) and then distributed via ${ }^{1} \mathrm{H}-{ }^{1} \mathrm{H}$ spin diffusion. Thus, the overall DNP efficiency is inevitably dependent on $T_{1 \mathrm{H}}$, against which the hyperpolarization is relayed through, i.e. "diffusion-limited".

The enhancement factors previously reported at $\sim 100 \mathrm{~K}$ for biological and pharmaceutical samples (Table S1) shows that for a range of samples there exists a tangible correlation between the maximum achievable enhancement and the number of methyl groups per molecular unit, such as amino-acid residue, which we call as the methyl-group density $\left(\rho\left(\mathrm{CH}_{3}\right)\right)$. In particular, crystalline samples seem to be strongly diffusion-limited due to its high (methyl-bearing) target-molecular density than dissolved systems, and larger thickness of domains in the order of $\mu \mathrm{m}$ than e.g. protein fibrillar assemblies with its cross-section in low nm-scale. Indeed, as we found at $16.4 \mathrm{~T}$, microcrystalline sample of a tri-peptide MLF (N-formyl Met-Leu-Phe-OH), a popular reference sample used in many MAS NMR experiments, ${ }^{4,5}$ yields no enhancement at $100 \mathrm{~K}$ due to its high methyl density $\rho\left(\mathrm{CH}_{3}\right)=1.0$. These observations point to the importance of polarization relay in making DNP more efficient in real applications.

While perdeuteration and amide ${ }^{1} \mathrm{H}$ back-exchange increases $T_{1 \mathrm{H}}$ and thus the hyperpolarization relay ${ }^{6,7}$, the required unfolding-refolding process, can be prohibitively difficult for membrane proteins. Deuteration also reduces sidechain resonances or its sensitivity essential for structural elucidation. Partial/fractional deuteration ${ }^{8}$ preserves protonated methyl groups $\left(\mathrm{CHD}_{2}\right.$ and $\mathrm{CH}_{3}$ ), and thus hardly benefits the DNP efficiency for diffusion-limited systems.

Here, we show at $16.4 \mathrm{~T}$ that ultra-low temperature (ULT; $T$ $\ll 100 \mathrm{~K})$ MAS DNP dramatically improve the signal enhancement in one of the most strongly diffusion-limited systems, microcrystalline MLF. As will be shown below, methyl-group rotation is attenuated at ULT, which as a result elongates $T_{1 \mathrm{H}}$ by a factor of $\sim 8$, promoting the polarization relay into the crystal. Requiring no modification to the sample, the ULT-DNP is a highly generic approach compatible with a wide range of samples. 

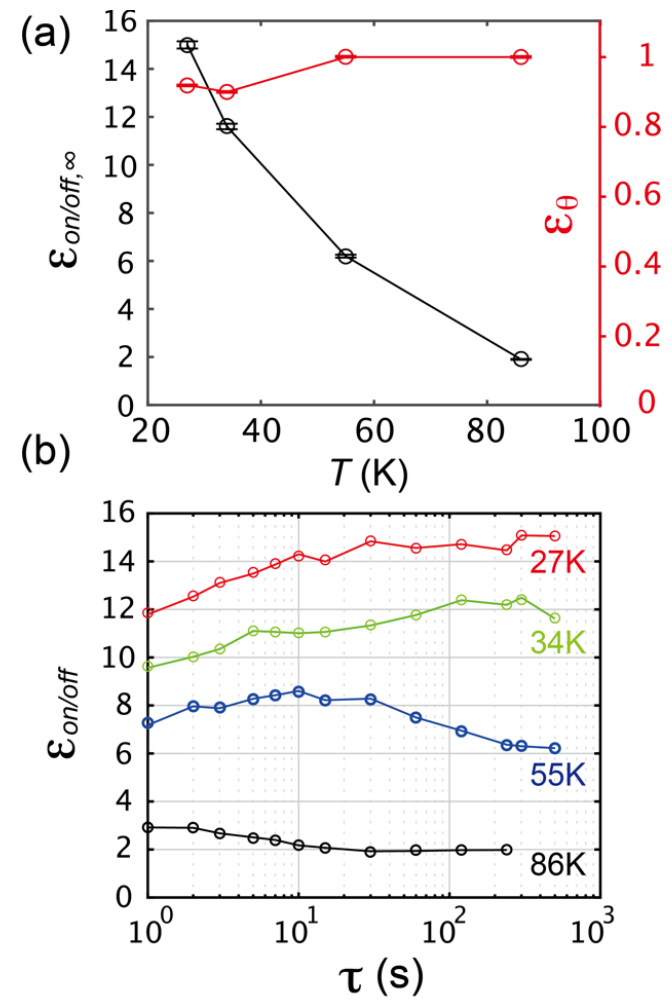

Figure 1 (a) Temperature dependence of the steady-state DNP enhancement factor $\epsilon_{\text {on } / \text { off }, \infty}$ (black, left axis) and paramagnet-induced signal loss $\epsilon_{\vartheta}$ (red, right axis) observed for microcrystalline MLF sample. (b) Enhancement as a function of the polarization buildup delay $\tau$ (in $\log$ scale) measured at indicated temperature. Fig. S2 shows the same data in a linear scale.

Fig.1a plots the steady-state enhancement $\epsilon_{\text {on } / \mathrm{ff}, \mathrm{\infty}}$ obtained for microcrystalline MLF wet with $20 \mathrm{mM}$ AMUPol-doped glycerol matrix $\left(\mathrm{d}_{8}\right.$-glycerol/ $\left.\mathrm{D}_{2} \mathrm{O} / \mathrm{H}_{2} \mathrm{O}=6 / 3 / 1 ; \mathrm{w} / \mathrm{w} / \mathrm{w}\right)$. Here, $\epsilon_{\text {on/off }}$ is the ratio of the signal intensity recorded with and without microwave (MW) irradiation. While the enhancement was very small $\left(\epsilon_{\mathrm{on} / \mathrm{of}, \infty}=1.9\right)$ at $86 \mathrm{~K}$ (and almost no enhancement at 100 $\mathrm{K})$, it steeply increased with lowering temperature, reaching 15 at $27 \mathrm{~K}$. This $\sim 8$-fold increase is attributed to the increase in the source polarization as well as the polarization-penetration depth into the crystal. The enhancement for a fully protonated protein (lysozyme) crystal was $\epsilon_{\text {on/of }} \sim 150$ at ULT (Fig. S1), being comparable to that reported for a perdeuterated protein crystal (SH3) at $\sim 100 \mathrm{~K}, 9.4 \mathrm{~T}^{6}{ }^{6}$ Also shown in Fig. 1a (red data) is the signal loss factor $\epsilon_{\vartheta}$ due to the paramagnetic quenching and MASinduced depolarization effects. The factor showed only $\sim 8 \%$ drop from $\sim 1.0$ at $86 \mathrm{~K}$ to $\sim 0.94$ at $27 \mathrm{~K}$; even if the radical resides on the crystal surface, the signal quenching only affects $<10 \mathrm{~nm}$, and the depolarization in the order of $100 \mathrm{~nm}$ from the surface.

Fig. 1b shows the enhancement factor during the polarization buildup, $\epsilon_{\text {on/off }}(\tau)=I_{\text {on }}(\tau) / I_{\text {off }}(\tau)$, where $I_{\text {on/off }}(\tau)$ is the signal intensity measured at delay $\tau$ with or without MW-irradiation. The value showed at early buildup time the transient enhancements higher ( $86 \mathrm{~K}$ and $55 \mathrm{~K}$ ) or lower $(34 \mathrm{~K}$ and $27 \mathrm{~K})$ than the steady state value $\left(\epsilon_{\text {on } / \mathrm{f}, \infty}\right)$. This is because the early buildup of the MW-on signal was faster or slower than the MW-off signal, respectively, characteristic to the DNP on heterogeneous systems. ${ }^{9}$ The change of the buildup profile over the temperature drop seems to correspond to a gradual broadening of the parameter-transition boundary ${ }^{9}$ between the polarization source (glycerol matrix) and the target (MLF crystal) due to the enlarging diffusion depth at lower temperatures, but needs further study to conclude.
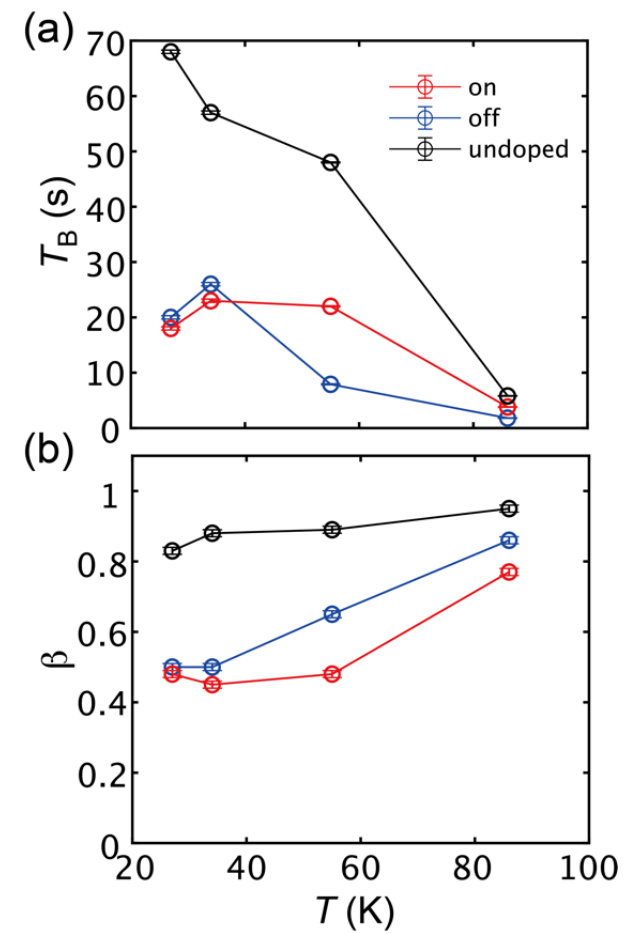

Figure 2 Temperature-dependence of the polarization buildup-time constant $T_{\mathrm{B}}$ (a) and the stretched exponents $\beta(\mathrm{b})$. Data are obtained with (red) and without (blue) MW-irradiation, and for an undoped sample (black).

Due to the inhomogeneity of the system, the polarization buildup curves for $I_{\mathrm{on}}(\tau)$ and $I_{\text {off }}(\tau)$ exhibited a multi-exponential feature, which we fitted using a stretched exponential function (Fig. S3): $I(\tau)=I_{\infty}\left(1-\exp \left[-\left(\tau / T_{\mathrm{B}}\right)^{\beta}\right]\right)$. Here, $I_{\infty}$ is the steady state intensity, $T_{\mathrm{B}}$ the effective buildup time constant and $\beta$ is the stretching exponent. The parameters $T_{\mathrm{B}}$ and $\beta$ obtained from the fit are shown in Fig. 2. At $86 \mathrm{~K}$, the polarization has grown nearly mono-exponentially $(\beta=0.8-0.9)$ with a small time-constant $T_{\mathrm{B}} \sim 1.8$ and $3.8 \mathrm{~s}$ for $I_{\text {on }}(\tau)$ and $I_{\text {off }}(\tau)$, respectively. From the short mono-exponential buildup and the small enhancement, it is suggested that at this relatively high temperature, $T_{1 \mathrm{H}}$ for MLF has a dominant contribution to the buildup, while the spin diffusion polarizes only a small layer beneath the crystal surface within the time window of $T_{\mathrm{B}} \sim 1.8 \mathrm{~s}$. Conversely, at $T<60 \mathrm{~K}$ the buildups for $I_{\text {on }}(\tau)$ and $I_{\text {off }}(\tau)$ exhibited a clear multi-exponential feature with $\beta=0.65$ at $55 \mathrm{~K}$ and 0.50 at 27 $34 \mathrm{~K}$, and a much larger time-constant, $T_{\mathrm{B}} \sim 8 \mathrm{~s}$ at $55 \mathrm{~K}$ and 20 $25 \mathrm{~s}$ at $27-34 \mathrm{~K}$. These intermediate $\beta$ near 0.5 indicates that the buildup has now been significantly affected by both the polarization relay from the surface and the intrinsic relaxation within the crystal. The time constant $T_{\mathrm{B}}$ showed 11-14-fold increase with the temperature drop due to gradual arrest of the methyl-group rotations at $<60 \mathrm{~K}$ (Fig. S4). This corresponds to the loss of unit-time sensitivity by a factor of 3.3-3.8, which is smaller than the overall signal enhancement, $\epsilon_{\mathrm{on} / \mathrm{fof}}=15$.

At $27 \mathrm{~K}$, the buildup of $I_{\text {on }}(\tau)$ could be fitted with a tripleexponential function with a similar least-square residual for the stretched exponential function (Fig. S3). The faster two buildup 
components had a [time constant, weight] of [ 1.9 s, 0.29] and $[\sim 16 \mathrm{~s}, 0.43]$ and the longest one $[>100 \mathrm{~s}, 0.28]$. It seems that the fastest-building component corresponds to the rapid outersurface penetration of the polarization identified at $86 \mathrm{~K}$ while the second-fast component may be attributed to an additional inner-surface penetration; the slowest one must be the longitudinal relaxation of the bulk crystal. From this time window of $\sim 16 \mathrm{~s}$ allowed for the penetration and assuming the average spin-diffusion rate of $D=1 \mathrm{~nm}^{2} / \mathrm{ms}$ typical to protonated organic polymers, the diffusion length $\sqrt{D t}$ from the crystal surface is estimated to be $\sim 130 \mathrm{~nm}$. Approximating each crystallite as a sphere with the observed diameter of $\sim 5 \mu \mathrm{m}$, the bulk enhancement of 15 obtained at $27 \mathrm{~K}$ (inner-rotor temperature of $\sim 40 \mathrm{~K}$ under MW irradiation) can be converted to the average surface ${ }^{1} \mathrm{H}$ polarization of $\sim 3.8 \%$ (Fig. S5).

To bear out the estimation experimentally, we directly measured the absolute ${ }^{1} \mathrm{H}$ polarization at the surface using the new method we recently developed (Fig. S6) ${ }^{10}$ as $3.1 \pm 0.1 \%$, which is not far away from the above estimation $\sim 3.8 \%$ based on a simple sphere model. This strengthen our view that the crystal surface is highly polarized without problem, while the limited diffusion into the crystal has made the bulk enhancement relatively small.

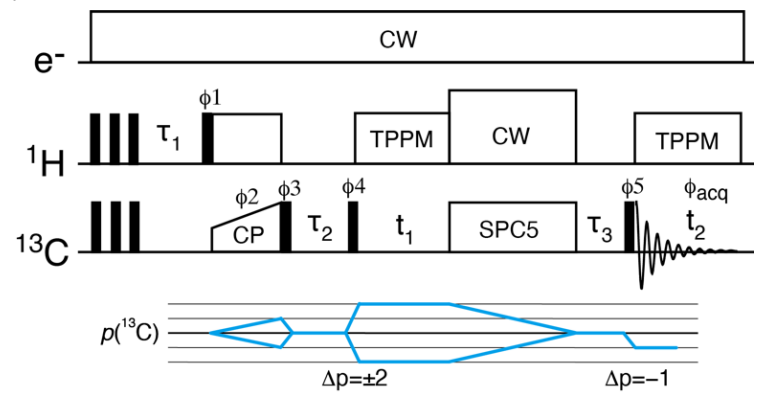

Figure 3 Pulse sequence for 2D surface-only DQ-SQ correlation experiment. $\tau_{1}=30 \mathrm{~s}, \tau_{2}=10 \mathrm{~ms}, \tau_{3}=22.7 \mu \mathrm{s}$. Rotor-synchronized element-pulse permutation was employed during the $t_{1}$ evolution. ${ }^{11}$ Other parameters are given in Supporting Information.

With the hyperpolarized crystal surface, we here demonstrate that it is possible to perform surface-only DNP-NMR spectroscopy, rather than the currently popular surface-enhanced DNP (SENS). ${ }^{12}$ The latter technique can suffer from background signals from the bulk that often cover up the interested spectral regions from the surface when both regions contain nuclei to be observed. In contrast, selective observation of the hyperpolarized domain without background contamination will prove to be useful in a range of applications. An attempt to a selective measurement using an extremely short DNP buildup time usually suffers from insufficient contrast between the interested and uninterested regions, especially when the latter is large in volume. In the pulse sequence in Fig. 3, the homonuclear twospin order $\left(2 S_{1 z} S_{2 z}\right)$ between ${ }^{13} \mathrm{C}$ spins is generated at the crystal surface after the $\left\{{ }^{1} \mathrm{H}\right\}-{ }^{13} \mathrm{C}$ cross-polarization (CP) from the hyperpolarized ${ }^{1} \mathrm{H}$ spins. This was directly converted to the multiple-quantum coherence $\left(2 S_{1 y} S_{2 y}\right)$ with a single non-selective ${ }^{13} \mathrm{C}$ $90^{\circ}$-pulse labeled with $\varphi 4$. In this work we selected the doublequantum (DQ) coherence by phase cycling of the latter pulse, evolved it during $t_{1}$, and then converted it back to the observable magnetization using the $\mathrm{DQ}{ }^{13} \mathrm{C}-{ }^{13} \mathrm{C}$ dipolar Hamiltonian recoupled using a symmetry sequence $\left(\mathrm{SPC}^{13}\right)$ before the signal acquisition. Due to the distinct operator form of the surface-excited two-spin order $\left(2 S_{1 z} S_{2 z}\right)$ to the Zeeman term $\left(S_{1 z}\right.$ and $\left.S_{2 z}\right)$, the DQ coherence selection thoroughly filters out the huge background signal from the crystal core (Fig. S7).

Fig 4 compares a high-field region of the surface-only and conventional 2D DQ-SQ spectra recorded with the same MLF sample under same measurement condition. It is intriguing to see that some of the peaks in the surface-only data showed significant peak shifts and/or broadening relative to the conventional data. Most prominent change was for $\delta_{\mathrm{DQ}}\left(\mathrm{C}^{\alpha}+\mathrm{C}^{\beta}\right)$ and $\delta_{\mathrm{SQ}}\left(\mathrm{C}^{\beta}\right)$ of Phe, showing upfield shifts by 0.98 and $0.82 \mathrm{ppm}$, respectively, indicated by arrows in Fig. $4 \mathbf{a}$; a slight line broadening was also observable for the $\delta_{\mathrm{DQ}}\left(\mathrm{C}^{\alpha}+\mathrm{C}^{\beta}\right)-\delta_{\mathrm{SQ}}\left(\mathrm{C}^{\beta}\right)$ peak. These site-specific peak shifts are more easily appreciated in 1D data, the Fourier transform of the first FID taken from the 2D DQ-SQ dataset, as compared with a regular CP spectrum (Fig. S8 and Table S2). Relatively large shifts with $\delta>0.3 \mathrm{ppm}$ were found for Met and Phe sidechains and Met $\mathrm{C}^{\circ}$ while none of such peak shifts were seen for the conventional 2D DQ-SQ data (Fig. S9). While it would be difficult to interpret these peak shifts as differential peptide conformation quantitatively, it may suggest a slight loosening or difference in crystal strain in the surface region of the crystal, leading to a distinct averaging of the chemical shifts that fluctuates on fs time-scale as has been suggested in simulations on MLF crystal. ${ }^{14}$ Since the peptide conformation of MLF in the crystal is known to be tightly bent at Leu with Met and Phe residues are closely packed to each other, it is reasonable that the conformational perturbation is correlated between these residues. ${ }^{5,14}$

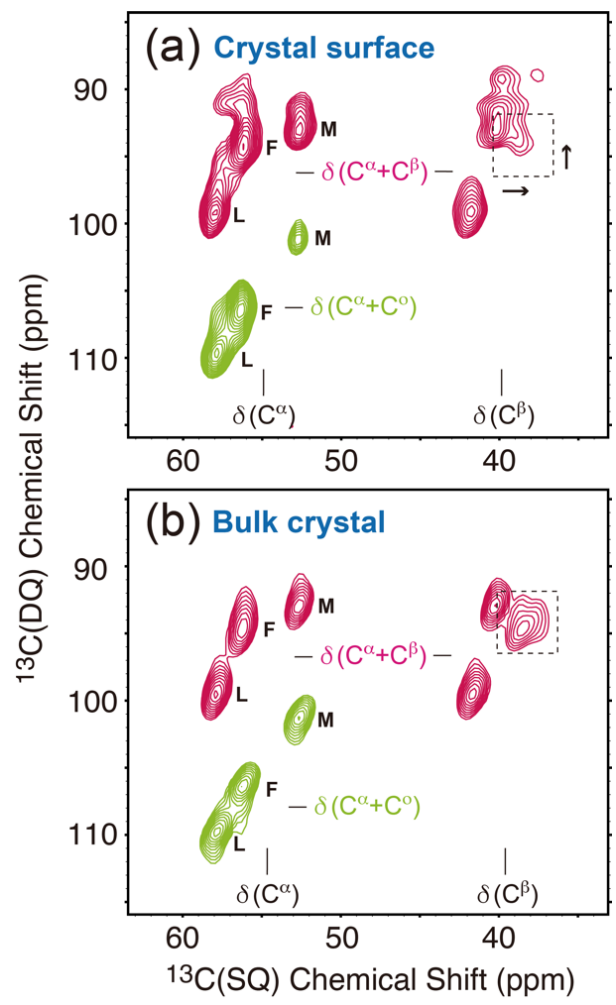

Figure 4 A high-field region of the surface-only (a) and conventional (b) 2D DQ-SQ correlation spectra for a microcrystalline MLF with DNP. $\mathrm{C}^{\alpha}-\mathrm{C}^{0}$ peaks are inverted due to aliasing; red and green contours show positive and negative peaks, respectively. Site-specific peak shifts are indicated with arrows in (a). M: Met, L: Leu and F: Phe.

The DQ-SQ peak intensities found in the surface-only data were $3.6 \%-4.0 \%$ of those in the regular DNP-enhanced DQ- 
SQ data for the bulk crystal, which is not far away from theoretical 5.3\% (Fig. S7). Although the surface-only data is $\sim 20$ fold less sensitive than the DNP-enhanced conventional DQ-SQ data, it may be accepted as a cost for the site-selective observation, also considering the interested surface volume is only $\sim 15 \%$ of the bulk crystal. Higher sensitivity should be achievable with higher enhancement using better polarizing agent, lower temperature, smaller crystalline size and/or more scans. With increased sensitivity, surface-specific structure measurements may also be possible, which is left for the future study. Similar site-selective experiment should be possible also at $\sim 100 \mathrm{~K}$ as long as the local polarization in the interested region of the system exceeds roughly $3 \%$ with e.g. $\epsilon_{\text {on/off }}>300$ at $100 \mathrm{~K}$, $9.4 \mathrm{~T}$.

In conclusion, ULT MAS capability provides a general means for improving the high-field DNP in diffusion-limited systems by increasing the diffusion length of the hyperpolarization. The two-spin order generated at a hyperpolarized domain enables spatially-selective spectroscopy which we demonstrated using microcrystalline MLF sample. The surface-only spectroscopy should greatly advance studies focused on polymer surfaces and extending tip of amyloid fibers, and by enabling e.g. organelle-selective spectroscopy in cell.

\section{ASSOCIATED CONTENT}

\section{Supporting Information}

The Supporting Information is available free of charge on the ACS Publications website: Additional experimental details and data on ultra-low temperature dynamic nuclear polarization-enhanced MAS NMR spectroscopy. (PDF)

\section{AUTHOR INFORMATION}

\section{Corresponding Author}

*tfjwr@protein.osaka-u.ac.jp

Notes

The authors declare no competing financial interest.

\section{ACKNOWLEDGMENT}

This work was supported by JST SENTAN (H271030 and JPMJSN15A1), the Ministry of Education, Culture, Sports, Science and Technology (MEXT), Japan (480790) (Y.M., T.F.).

\section{REFERENCES}

(1) Lilly Thankamony, A. S.; Wittmann, J. J.; Kaushik, M.; Corzilius, B. Dynamic Nuclear Polarization for Sensitivity Enhancement in Modern Solid-State NMR. Prog. Nucl. Magn. Reson. Spectrosc. 2017, 102-103, 120-195. https://doi.org/10.1016/j.pnmrs.2017.06.002.

(2) Geiger, M.-A.; Orwick-Rydmark, M.; Märker, K.; Franks, W. T.; Akhmetzyanov, D.; Stöppler, D.; Zinke, M.; Specker, E.; Nazaré, M.; Diehl, A.; van Rossum, B.-J.; Aussenac, F.; Prisner, T.; Akbey, Ü.; Oschkinat, H. Temperature Dependence of Cross-Effect Dynamic Nuclear Polarization in Rotating Solids: Advantages of Elevated
Temperatures. Phys. Chem. Chem. Phys. 2016, 18 (44), 30696-30704. https://doi.org/10.1039/c6cp06154k.

(3) Rosay, M.; Blank, M.; Engelke, F. Instrumentation for Solid-State Dynamic Nuclear Polarization with Magic Angle Spinning NMR. J. Magn. Reson. 2016, 264, 88-98. https://doi.org/10.1016/j.jmr.2015.12.026.

(4) Rienstra, C. M.; Hohwy, M.; Hong, M.; Griffin, R. G. 2D and $3 \mathrm{D}{ }^{15} \mathrm{~N}-{ }^{13} \mathrm{C}-{ }^{13} \mathrm{C}$ NMR Chemical Shift Correlation Spectroscopy of Solids : Assignment of MAS Spectra of Peptides. 2000, 115 (No. 14), 10979-10990.

(5) Bajaj, V. S.; Wel, P. C. A. Van Der; Griffin, R. G. Observation of a Low-Temperature, Dynamically Driven Structural Transition in a Polypeptide by Solid-State NMR Spectroscopy. 2009, 125 (15), 118-128.

(6) Akbey, Ü.; Franks, W. T.; Linden, A.; Lange, S.; Griffin, R. G.; Van Rossum, B. J.; Oschkinat, H. Dynamic Nuclear Polarization of Deuterated Proteins. Angew. Chemie - Int. Ed. 2010, 49 (42), 78037806. https://doi.org/10.1002/anie.201002044.

(7) Koers, E. J.; Van Der Cruijsen, E. A. W.; Rosay, M.; Weingarth, M.; Prokofyev, A.; Sauvée, C.; Ouari, O.; Van Der Zwan, J.; Pongs, O.; Tordo, P.; Maas, W. E.; Baldus, M. NMR-Based Structural Biology Enhanced by Dynamic Nuclear Polarization at High Magnetic Field. J. Biomol. NMR 2014, 60 (2-3), 157-168. https://doi.org/10.1007/s10858-014-9865-8.

(8) Nand, D.; Cukkemane, A.; Becker, S.; Baldus, M. Fractional Deuteration Applied to Biomolecular Solid-State NMR Spectroscopy. J. Biomol. NMR 2012, 52 (2), 91-101. https://doi.org/10.1007/s10858011-9585-2.

(9) Pinon, A. C.; Schlagnitweit, J.; Berruyer, P.; Rossini, A. J.; Lelli, M.; Socie, E.; Tang, M.; Pham, T.; Lesage, A.; Schantz, S.; Emsley, L. Measuring Nano- to Microstructures from Relayed Dynamic Nuclear Polarization NMR. J. Phys. Chem. C 2017, 121 (29), 15993-16005. https://doi.org/10.1021/acs.jpcc.7b04438.

(10) Sugishita, T.; Matsuki, Y.; Fujiwara, T. Absolute ${ }^{1} \mathrm{H}$ Polarization Measurement with a Spin-Correlated Component of Magnetization by Hyperpolarized MAS-DNP Solid-State NMR. Solid State Nucl. Magn. Reson. 2019, 99 (February), 20-26. https://doi.org/10.1016/j.ssnmr.2019.02.001.

(11) Hong, M. Solid-State Dipolar INADEQUATE NMR Spectroscopy with a Large Double-Quantum Spectral Width. J. Magn. Reson. 1999, 136 (1), 86-91. https://doi.org/10.1006/jmre.1998.1631.

(12) Pump, E.; Bendjeriou-Sedjerari, A.; Viger-Gravel, J.; Gajan, D.; Scotto, B.; Samantaray, M. K.; Abou-Hamad, E.; Gurinov, A.; Almaksoud, W.; Cao, Z.; Lesage, A.; Cavallo, L.; Emsley, L.; Basset, J. M. Predicting the DNP-SENS Efficiency in Reactive Heterogeneous Catalysts from Hydrophilicity. Chem. Sci. 2018, 9 (21), 4866-4872. https://doi.org/10.1039/c8sc00532j.

(13) Hohwy, M.; Rienstra, C. M.; Jaroniec, C. P.; Griffin, R. G.; Introduction, I. Fivefold Symmetric Homonuclear Dipolar Recoupling in Rotating Solids: Application to Double Quantum Spectroscopy. J. Chem. Phys.m 1999, 110 (16), 7983-7992.

(14) Gortari, I. De; Portella, G.; Salvatella, X.; Bajaj, V. S.; Wel, P. C. A. Van Der; Yates, X. J. R.; Segall, M. D.; Pickard, C. J.; Payne, M. C.; Vendruscolo, M. Time Averaging of NMR Chemical Shifts in the MLF Peptide in the Solid State. J. Am. Chem. Soc. 2010, No. 10, 5993-6000. 
TOC figure

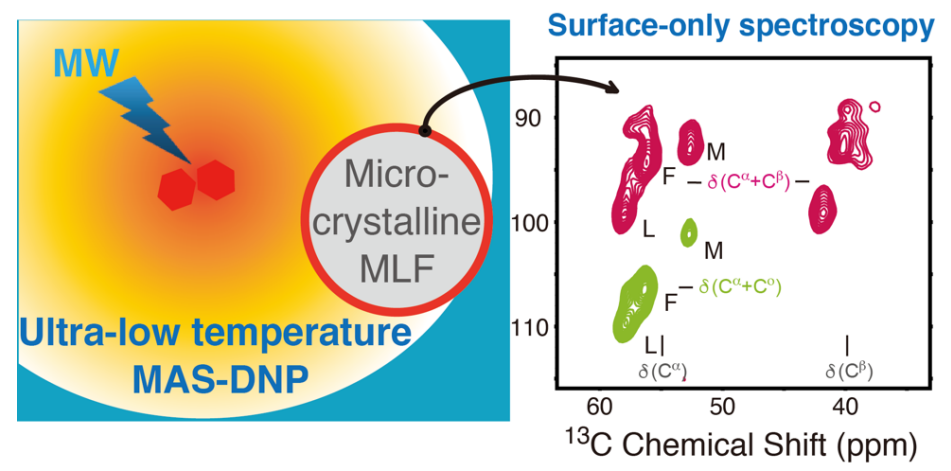

